\title{
Inertial endomorphisms of an abelian group
}

\author{
Ulderico Dardano • Silvana Rinauro
}

Received: 15 October 2013 / Accepted: 8 October 2014 / Published online: 21 October 2014

(C) Fondazione Annali di Matematica Pura ed Applicata and Springer-Verlag Berlin Heidelberg 2014

\begin{abstract}
We describe inertial endomorphisms of an abelian group $A$, that is endomorphisms $\varphi$ with the property $|(\varphi(X)+X) / X|<\infty$ for each $X \leq A$. They form a ring $I E(A)$ containing the ideal $F(A)$ formed by the so-called finitary endomorphisms, the ring of power endomorphisms and also other non-trivial instances. We show that the quotient ring $I E(A) / F(A)$ is commutative. Moreover, inertial invertible endomorphisms form a group, provided $A$ has finite torsion-free rank. In any case, the group $\operatorname{IAut}(A)$ they generate is commutative modulo the group $F \operatorname{Aut}(A)$ of finitary automorphisms, which is known to be locally finite. We deduce that $\operatorname{IAut}(A)$ is locally-(center-by-finite). Also, we consider the lattice dual property, that is $|X /(X \cap \varphi(X))|<\infty$ for each $X \leq A$ and show that this implies the above one, provided $A$ has finite torsion-free rank.
\end{abstract}

Keywords Inertial groups $\cdot$ Inert subgroup $\cdot$ Finitary automorphism $\cdot$ Power endomorphism $\cdot$ Locally finite group

Mathematics Subject Classification Primary 20K30; Secondary 20E07 · 20E36 2 20F24

\section{Introduction}

Recently, there has been interest for totally inert (TIN) groups, i.e., groups whose all subgroups are inert (see $[1,5,8,11]$ ). A subgroup is said inert if it is commensurable to each

Dedicated to H. Heineken.

U. Dardano $(\varangle)$

Dipartimento di Matematica e Applicazioni “R.Caccioppoli”, Università di Napoli

"Federico II", Via Cintia - Monte S. Angelo, 80126 Naples, Italy

e-mail: dardano@unina.it

S. Rinauro

Dipartimento di Matematica, Informatica ed Economia, Università della Basilicata,

Via dell'Ateneo Lucano 10, 85100 Potenza, Italy

e-mail: silvana.rinauro@unibas.it 
conjugate of its. Two subgroups $X, Y$ of any group are told commensurable iff $X \cap Y$ has finite index in both $X$ and $Y$ (see [12]).

When dealing with soluble TIN-groups one is concerned with automorphisms with the following property. As in [6] and [7], an endomorphism $\varphi$ of an abelian group A (from now on always in additive notation) is said (right-) inertial iff:

$$
\forall X \leq A|\varphi(X)+X / X|<\infty .
$$

Consideration of endomorphisms instead of automorphisms only is due to the fact we state below. Moreover, notice that in [7] the concept of inertial endomorphism is related to the investigation of the dynamical properties of an endomorphism of an abelian group.

Fact Inertial endomorphisms of any abelian group A form a ring, say IE(A), containing the ideal $F(A)$ of endomorphisms with finite image.

To prove this notice that if both $\varphi_{1}$ and $\varphi_{2}$ are inertial endomorphisms of $A$ (that is have the above RIN), then $\forall X \leq A \quad\left|\left(X+\varphi_{1}(X)\right) / X\right|<\infty$ and $\mid\left(X+\varphi_{1}(X)+\varphi_{2}(X)+\right.$ $\left.\varphi_{1} \varphi_{2}(X)\right) /\left(X+\varphi_{1}(X)\right) \mid<\infty$.

In this paper, by our main result Theorem A below, we give a characterization of inertial endomorphisms of an abelian group, from which we deduce useful consequences. In particular, we have:

Corollary A The ring $I E(A) / F(A)$ is commutative.

In Proposition A, we will exhibit non-trivial instances of inertial endomorphisms. For details on the additive group of $I E(A)$ see [4].

As far as invertible inertial endomorphisms concern, recall that in [2] one finds a characterization of groups of automorphisms $\Gamma \leq \operatorname{Aut}(A)$ with the property that for each subgroup $X \leq A$ there is a $\Gamma$-invariant subgroup $X^{\Gamma} \geq X$ such that $\left|X^{\Gamma}: X\right|$ is finite. Later in [9], a characterization when $\Gamma$ has the dual property, that there is a $\Gamma$-invariant subgroup $X_{\Gamma} \leq X$ such that $\left|X: X_{\Gamma}\right|$ is finite, was given. Clearly, in both cases, $\Gamma$ is formed by automorphisms which are inertial. Then, in [3], we put both pictures in the same framework by characterizing finitely generated groups $\Gamma$ of automorphisms which are inertial and have inertial inverse. In Propositions 2.2 and 2.3 of this paper, we give a characterization also in the more general setting of endomorphisms.

Consideration of the map $x \mapsto 2 x$ shows that the inverse of an inertial automorphism of $\mathbb{Q}^{\omega}$ need not to be inertial. However, the inverse $\varphi$ of an inertial invertible endomorphism has the lattice dual property (say left-inertial) that is:

$$
\forall X \leq A|X /(X \cap \varphi(X))|<\infty .
$$

On the other hand, from results in [3], we have that for an automorphism $\varphi$ of a periodic abelian group $A$ properties LIN and RIN (i.e., inertial) are equivalent, that is each subgroup is commensurable with its image. Therefore, in [3] by inertial we meant LIN and RIN, while here by inertial we mean just RIN. Next, corollary to Theorem A below investigates how these properties are related. Then, we consider the group, say I Aut $(A)$, generated by inertial automorphisms of an abelian group A. Notice that I Aut $(A)$ contains the group of so-called almost-power automorphisms, that is $\gamma \in \operatorname{Aut}(A)$ such that each subgroup of $A$ contains a $\gamma$-invariant subgroup of finite index. This latter group has been introduced in [9].

Recall that the rank $r_{0}(A)$ of any free abelian subgroup $F$ of $A$ such that $A / F$ is periodic is said torsion-free rank of $A$. 
Corollary B Let $\varphi$ be an endomorphism of an abelian group A.

(1) If $r_{0}(A)<\infty$, then LIN implies RIN (i.e., inertial) and the two properties are equivalent if $\varphi$ is an automorphism. Thus, inertial automorphisms form the group I Aut (A).

(2) If $r_{0}(A)=\infty$, then I Aut $(A)$ is formed by the products $\gamma_{1} \gamma_{2}^{-1}$, where $\gamma_{1}, \gamma_{2}$ are both inertial automorphisms.

From Proposition 2.3, we will also have that when $r_{0}(A)=\infty$ an endomorphism is both LIN and RIN iff it acts as the identity or the inversion map on a subgroup with finite index.

Recall that, automorphisms acting as the identity map on a finite index subgroup form a group, F Aut (A), which is locally finite (see [13]). Clearly, F Aut (A) $\leq \operatorname{IAut}(A)$. Actually, I Aut $(A)$ need not to be periodic, but its periodic elements form a subgroup containing the derived subgroup as by next statement, which also follows from Theorem A.

Theorem B Let $\Gamma=I$ Aut $(A)$ be the group generated by the inertial automorphisms of an abelian group A. Then:

(1) $\Gamma^{\prime} \leq F A u t(A)$ is locally finite;

(2) $\Gamma$ is locally central-by-finite.

Note that there are non-elementary instances of periodic non-finitary inertial automorphisms. To see this, consider the $p$-group $B \oplus D$ where $(p \neq 2) B$ is infinite bounded and $D$ is divisible with finite rank and the automorphism acting as the identity on $B$ and the inversion map on $D$. On the other hand, the abelian group $\operatorname{IAut}(A) / F A u t(A)$ may be rather large as we see in next statement.

Proposition A There exists a countable abelian group A with $r_{0}(A)=1$ such that I Aut $(A)$ has a subgroup $\Sigma \simeq \prod_{p} \mathbb{Z}(p)$ with $\Sigma \cap F$ Aut $(A)=T(\Sigma) \simeq \bigoplus_{p} \mathbb{Z}(p)$, where p ranges over the set of all primes.

In this first section, we have focused on applications of our main results, which will be stated in next Sect. 2, where we also introduce some terminology and definitions before. In Sect. 3, we prove some preliminary facts, while in Sect. 4, we handle periodic abelian groups. Section 5 is devoted to the general case, and in the final Sect. 6, we give the proof of the main Theorem A and consequences of its stated in this section.

\section{Statements of main results}

As a standard reference on abelian groups we use [10]. Letter $A$ always denotes an abelian group, we regard as a left $E(A)$-module, where $E(A)$ denotes the ring of endomorphism of $A$. Any $p$-group is regarded as a module over the ring $\mathbb{J}_{p}$ of $p$-adics as well. Letters $\varphi, \gamma$ denote endomorphisms of $A$, while $m, n, r, s, t$ denote integers, $p$ a prime, $\pi$ a set of primes, $\varpi(n)$ the set of prime divisors of $n$ and $\varpi(A)$ the set of primes $p$ such that there is an element of $A$ with order $p$. We denote by $T=T(A)$ the torsion subgroup of $A$, by $A_{\pi}$ the $\pi$-component of $A$, by $A[n]:=\{a \in A \mid n a=0\}$. If $n A=0$ and $n \neq 0$, we say that $A$ is bounded by $n$. Further, we say that $A$ is bounded, if it is bounded by some $n$. We say that $A$ is $\pi$-divisible when $p A=A$ for each $p \in \pi$ and denote by $\operatorname{Div}(A)$ the largest subgroup of $A$ which is divisible by each prime. As usual $\mathbb{Q}^{\pi}$ denotes the ring of rationals whose denominator is a $\pi$-number, where $\mathbb{Q}^{\varpi(n)}=\mathbb{Z}\left[\frac{1}{n}\right]$. When we write $m / n \in \mathbb{Q}$, we always mean $m$ and $n \neq 0$ are coprime integers. 
Definition We say that an endomorphism $\varphi$ of an abelian group $A$ is a multiplication if one of the following holds:

(1) $A$ is periodic and $\varphi$ is a so-called power endomorphism, that is acting by a $p$-adic on each $p$-component of $A$. This is equivalent to saying that $\varphi$ leaves each subgroup invariant, i.e., $\forall X \leq A \varphi(X) \subseteq X$ (see [11]).

(2) $A$ is not periodic and there are $m, n \in \mathbb{Z}$ such that $A=n A, A_{\varpi(n)}=0$ and $\varphi(n x)=m x$ for all $x \in A$. We just write $\varphi=\frac{m}{n}$ as $A$ has a natural structure of $\mathbb{Q}^{\varpi(n)}$-module.

Note that we use word "multiplication" in a way different from [10]. Ours are in fact "scalar multiplications". When $A$ is periodic, multiplication are plainly inertial (otherwise see Proposition 3.2).

Fact Multiplications of an abelian group A form a ring $M(A)$ and commute with any endomorphism.

Recall that $\mathbb{J}_{p}$ contains the ring of rationals whose denominator is coprime to $p$. Further if $A$ is non-periodic, then $M(A) \simeq \mathbb{Q}^{\pi}$ where $\pi$ is the largest set of primes such that $A$ is a $\mathbb{Q}^{\pi}$-module, that is $A$ is $p$-divisible with no elements of order $p, \forall p \in \pi$.

If $X$ is a subset of a (left) $R$-module $A$ and $R_{1}$ a subring of $R$ as usual we denote by $\langle X\rangle$ (respectively, $R_{1} X$ or $X^{R_{1}}$ ) the additive subgroup (respectively, the $R_{1}$-submodule) spanned by $X$. Further, by $X_{R_{1}}$, we denote the largest $R_{1}$-submodule contained in $X$. Also if $\varphi$ an endomorphism of $A$ we write $X^{(\varphi)}$ for $\mathbb{Z}[\varphi] X$ and $X_{(\varphi)}$ for $X_{\mathbb{Z}[\varphi]}$. Note that, by abuse of notation, we sometimes identify $\mathbb{Z}$ (and even $\mathbb{Q}^{\pi}$ ) with their (possibly not faithful) natural images in $E(A)$.

Before we embark on the description of inertial endomorphisms, note a sufficient condition for an endomorphism to be inertial. We omit the straightforward proof.

Proposition 2.1 Let $\varphi$ be an endomorphism of an abelian group A. If $\varphi$ acts as an inertial (respectively, LIN) endomorphism either on a finite index subgroup of $A$ or modulo a finite subgroup, then $\varphi$ is inertial (respectively, LIN) on A.

We state now a detailed characterization of inertial endomorphisms of periodic abelian groups. We handle LIN endomorphisms as well.

Proposition 2.2 Let $\varphi_{1}, \ldots \varphi_{t}$ be finitely many endomorphisms of an abelian periodic group $A$ and $\Phi:=\mathbb{Z}\left[\varphi_{1}, \ldots \varphi_{t}\right] \leq E(A)$. Then:

$(R)$ each $\varphi_{i}$ is inertial iff there is a finite index subgroup $A_{0}=B \oplus D \oplus C$ of $A$ such that $B, C, D$ are $\Phi$-invariant and:

(i) $\varpi(B \oplus D) \cap \varpi(C)=\emptyset$,

(ii) $B$ is bounded and $D$ is divisible with Min,

(iii) each $\varphi_{i}$ acts as multiplication on $B, D$ and $C$.

If the above holds, then

$$
\exists m \forall X \leq A \quad\left|X^{\Phi} / X_{\Phi}\right| \leq m .
$$

(L) each $\varphi_{i}$ is LIN iff it is inertial and there are subgroups $A_{0}, B, D, C$ as in $(R)$ such that $\varphi_{i}$ acts as a nonzero multiplication on each nonzero primary component of $D$ and an invertible multiplication on $B$ and $C$.

We treat now inertial and LIN endomorphisms of a non-periodic abelian group. Next, proposition generalizes Theorem 3 of [3] to endomorphisms. Even if the statement has interest in itself, we will regard it as a lemma for Theorem A. 
Proposition 2.3 An endomorphism $\varphi$ of an abelian non-periodic group A is inertial (respectively, LIN) if and only if either (a) or (b) holds:

(a) there is a $\varphi$-invariant finite index subgroup $A_{0}$ of $A$ such that $\varphi$ acts as multiplication by $m \in \mathbb{Z}$ (by $\frac{1}{n} \in \mathbb{Q}$, respectively) on $A_{0}$;

(b) there are finitely many elements $a_{1}, \ldots, a_{r}$ such that:

(i) $\varphi$ acts as multiplication by $\frac{m}{n} \in \mathbb{Q}$ on the $\varphi$-submodule $V=\mathbb{Z}[\varphi]\left\langle a_{1}, \ldots, a_{r}\right\rangle$ which is torsion free as an abelian group,

(ii) the factor group $A / V$ is torsion and $\varphi$ induces an inertial (respectively, LIN) endomorphism on $A / V$,

(iii) the $\varpi(n)$-component of $A$ is bounded.

We state now our main result, which characterizes inertial endomorphims of any abelian group. Notice that when $A$ is periodic, the statement of Theorem A applies with $V=0$ and implies part (R) of Proposition 2.2. On the other hand, if $A$ is torsion free, then inertial endomorphisms are just multiplications, see Proposition 3.3. In fact, in the statement of Theorem A, we will have $\varphi_{i}=\frac{m_{i}}{n_{i}} \in \mathbb{Q}$ on $A / T(A)$ for each $i$.

Theorem A Let $\varphi_{1}, \ldots, \varphi_{t}$ be finitely many endomorphisms of an abelian group $A$ and $\Phi:=\mathbb{Z}\left[\varphi_{1}, \ldots \varphi_{t}\right] \leq E(A)$. Then, each $\varphi_{i}$ is inertial if and only if there is a $\Phi$-invariant subgroup $A_{0}$ with finite index in A such that either and (a) or (b) holds:

(a) each $\varphi_{i}$ acts as multiplication by $m_{i} \in \mathbb{Z}$ on $A_{0}$;

(b) there are $\Phi$-invariant subgroups $B, C, D$ of $A$ and finite sets of primes $\pi \subseteq \pi_{1}$ such that:

$$
A_{0}=B \oplus D \oplus C
$$

where

(i) $B \oplus D$ is the $\pi_{1}$-component of $A_{0}$ where $B$ is bounded and $D$ is a divisible $\pi^{\prime}$-group with finite rank,

(ii) $C$ is a $\mathbb{Q}^{\pi}\left[\varphi_{1}, \ldots, \varphi_{t}\right]$-module, with a submodule $V \simeq \mathbb{Q}^{\pi} \oplus \cdots \oplus \mathbb{Q}^{\pi}$ (finitely many times) such that $C / V$ is a $\pi_{1}$-divisible $\pi^{\prime}$-group,

(iii) each $\varphi_{i}$ acts as (possibly different) multiplications on $B, D, V, C / V$,

(iv) each $\varphi_{i}$ acts by the same $\frac{m_{i}}{n_{i}} \in \mathbb{Q}$ on $V$ and all $p$-components $D_{p}$ of $D$ with the property that the $p$-component of $C / V$ is infinite; also $\pi=\varpi\left(n_{1} \cdots n_{t}\right)$.

\section{Multiplications of an abelian group}

We start this section by pointing out which multiplications are inertial or LIN, respectively.

Recall that an abelian group $A$ with the minimal condition (Min) is just a group of the shape $A=F \oplus D$, where $F$ is finite and $D$ is divisible with finite total rank that is the sum of finitely many infinite cocyclic (Prüfer) groups.

Proposition 3.1 Let $A$ be an infinite abelian group and $\varphi \in M(A)$.

(R) If $A$ is periodic, then each multiplication is inertial;

(L) If $A$ is a $p$-group, then $\varphi$ is LIN iff $\varphi$ is invertible or A has Min and $\varphi \neq 0$.

Proof Part (R) is trivial. Concerning part (L), clearly $\varphi=0$ is not LIN as $A$ is infinite. Then, let the $p$-adic $\alpha=p^{s} \alpha_{1}$ represent $\varphi$ on $A$ with $\alpha_{1}$ invertible. If $\varphi$ is not invertible, then $s>0$ 
and $A[p] \leq \operatorname{ker} \varphi$ is finite. Hence, $A$ has Min. Conversely, if $A$ has Min, then for any $X \leq A$ we have that $X / \varphi(X)=X / p^{s} X$ is finite.

Recall that multiplications of a non-periodic abelian group are all of type $\varphi=\frac{m}{n} \in \mathbb{Q}$.

Proposition 3.2 Let $A$ be an abelian non-periodic group and $\varphi=\frac{m}{n} \in M(A)$.

(R) $\varphi$ is inertial iff either $r_{0}(A)<\infty$ or $n= \pm 1$;

(L1) if $0<r_{0}(A)<\infty$, then $\varphi$ is LIN iff $A_{\varpi(m)}$ has Min and $m \neq 0$;

(L2) if $r_{0}(A)=\infty$, then $\varphi$ is LIN iff $m= \pm 1$.

Proof (R) Arguing in $\bar{A}:=A / T(A)$, we have that for any $\bar{X} \leq \bar{A}$ free with infinite rank, it results that

$$
\frac{\varphi(\bar{X})+\bar{X}}{\bar{X}} \simeq \frac{m \bar{X}+n \bar{X}}{n \bar{X}}
$$

is infinite unless $n= \pm 1$. Then, if $\varphi$ is inertial and $r_{0}(A)=\infty$, we have that $\varphi$ is a multiplication by the integer $m$.

Conversely, if $n= \pm 1$, then $\varphi$ is trivially inertial. Assume $r_{0}(A)<\infty$. For any $X \leq A$, the section $(\varphi(X)+X) / X$ is a bounded $\varpi(n)$-group and is finite $\bmod T:=T(A)$. On the other hand, since $A$ is a $\mathbb{Q}^{\varpi(n)}$-module, $A_{\varpi(n)}=0$ and therefore $(\varphi(X)+X) / X$ avoids $T$.

(L1) If $\varphi$ is LIN, then $A / \varphi(A)<\infty$ implies that $\varphi \neq 0$. Further, we have that $A_{\varpi(m)}$ has Min, by Proposition 3.2. Conversely, for each $X \leq A$ we have

$$
\frac{X}{X \cap \frac{m}{n} X} \simeq \frac{n X}{n X \cap m X}
$$

is finite as it is bounded by $m$ and both the rank of $A_{\varpi(m)}$ and torsion-free rank of $A$ are finite.

(L2) Let $\bar{X}$ be free subgroup of $\bar{A}:=A / T$ with infinite rank. By the same argument as above, we have that $\bar{X} /\left(\bar{X} \cap \frac{m}{n} \bar{X}\right)$ is infinite unless $m= \pm 1$. Conversely, $\varphi=\frac{1}{n}$ is LIN as $X \leq \varphi(X)$ for each $X \leq A$.

The other way round, let us see that inertial endomorphisms of a torsion-free abelian group are all multiplications.

Proposition 3.3 Let $\varphi$ be an endomorphism of a torsion-free abelian group A.

(R) $\varphi$ is inertial iff $\varphi$ acts as a multiplication by $\frac{m}{n} \in \mathbb{Q}$ and if $r_{0}(A)=\infty$ then $n= \pm 1$.

(L) $\varphi$ is LIN iff $\varphi$ acts as a multiplication by $\frac{m}{n} \in \mathbb{Q}$ with $m \neq 0$ and if $r_{0}(A)=\infty$ then $m= \pm 1$.

In particular, if $\varphi \neq 0$ and $r_{0}(A)<\infty$, $\varphi$ is LIN iff $\varphi$ is inertial.

Proof The sufficiency of the conditions follows from Proposition 3.2. To prove necessity, we generalize an argument used in [3]. In both cases (R) and (L), for each $a \in A$ there exist nonzero $m, n \in \mathbb{Z}$ such that $m a=n \varphi(a)$. As $A$ is torsion free, $m, n$ can be choosen coprime. Let us show that $\frac{m}{n}$ is independent of $a$. Let $a_{1} \in A$. If $\left\langle a_{1}\right\rangle \cap\langle a\rangle \neq\{0\}$, then $k a_{1}=h a$ for some nonzero $h, k \in \mathbb{Z}$. Therefore, we may write $\varphi\left(a_{1}\right)=\frac{h}{k} \varphi(a)=\frac{h}{k} \frac{m}{n} a=\frac{m}{n} a_{1}$. Similarly, if $\left\langle a_{1}\right\rangle \cap\langle a\rangle=\{0\}$, there exist $m_{1}, m_{2}, n_{1}, n_{2} \in \mathbb{Z}$ such that

$$
\frac{m}{n} a+\frac{m_{1}}{n_{1}} a_{1}=\varphi(a)+\varphi\left(a_{1}\right)=\varphi\left(a+a_{1}\right)=\frac{m_{2}}{n_{2}}\left(a+a_{1}\right)=\frac{m_{2}}{n_{2}} a+\frac{m_{2}}{n_{2}} a_{1} .
$$

It follows $\frac{m}{n}=\frac{m_{2}}{n_{2}}=\frac{m_{1}}{n_{1}}$. Thus, $\varphi$ acts as a multiplication.

For the rank restriction, apply Proposition 3.2. 
We will use often the following fact. Even if it follows from Theorem 3 of [4] as a particular case, we sketch here the very elementary proof.

Proposition 3.4 For an endomorphism $\varphi$ of a periodic abelian group A the following are equivalent:

$(M F) \varphi$ acts as a multiplication on a finite index subgroup $A_{0}$ of $A$,

(FM) $\varphi$ acts as a multiplication modulo a finite subgroup $A_{1}$ of $A$.

Proof If $(M F)$ holds then $\varphi$ acts as a multiplication on all but finitely many components $A_{p_{1}}, \ldots, A_{p_{t}}$. If $A_{p_{i}}$ is any of them and the $p_{i}$-adic $\alpha_{i}$ represents $\varphi$ on $A_{0} \cap A_{p_{i}}$, then $B_{p_{i}}:=\left(\varphi-\alpha_{i}\right)\left(A_{0} \cap A_{p_{i}}\right)$ is an image of $A / A_{0}$. Thus, $A_{1}:=B_{p_{1}}+\cdots+B_{p_{t}}$ is the desired subgroup. The converse is similar.

FM-endomorphisms are inertial by Proposition 2.1 and will play a relevant role in the sequel.

\section{Inertial endomorphisms of a periodic abelian group}

This section is actually devoted to prove Proposition 2.2, which can be regarded as the periodic case of Theorem A. We state first the following easy but fundamental fact, which will reduce the proof to the case when $A$ is a $p$-group.

Proposition 4.1 An endomorphism of an abelian torsion group A is inertial (respectively, LIN) iff it is such on all primary components and multiplication (respectively, invertible multiplication) on all but finitely many of them.

Proof Sufficiency of the condition may be verified straightforward. Concerning necessity, we only deal with case LIN, the other case being similar. Let $\pi$ be the set of primes $p$ such that $\varphi$ is not an invertible multiplication on $A_{p}$. If $p \in \pi$, then either $\varphi$ is not a multiplication on $A_{p}$ or $\varphi$ is a non-invertible multiplication. In the former case, there is a cyclic subgroup $X_{p}$ of $A_{p}$ such that $\varphi\left(X_{p}\right) \nsubseteq X_{p}$, and hence $\left|X_{p} \cap \varphi\left(X_{p}\right)\right|<\left|\varphi\left(X_{p}\right)\right| \leq\left|X_{p}\right|$. In the latter case, there is a cyclic subgroup $X_{p}$ of $A_{p}$ such that $\varphi\left(X_{p}\right)$ is properly contained in $X_{p}$. In both cases $\left|X_{p} /\left(X_{p} \cap \varphi\left(X_{p}\right)\right)\right|>1$. It is now clear that if $\varphi$ is LIN, then $\pi$ is finite, as $|X /(X \cap \varphi(X))|$ must be finite for $X:=\bigoplus_{p \in \pi} X_{p}$.

We prove now a few lemmas. The first one extends Proposition 4.3 in [11].

Lemma 4.2 Let $A$ be an abelian p-group, $a \in A$ and $\varphi \in E(A)$.

(1) If $\varphi$ is either inertial or LIN, then a belongs to a finite $\varphi$-invariant subgroup.

(2) If $\left|X / X_{(\varphi)}\right|<\infty$ for all $X \leq A$, then $\left|X^{(\varphi)} / X\right|<\infty$ for all $X \leq A$.

(3) If $\left|X / X_{(\varphi)}\right| \leq p^{m}$ for all $X \leq A$, then $\left|X^{(\varphi)} / X\right| \leq p^{m^{2}}$ for all $X \leq A$.

Proof (1) We may assume $A=\langle a\rangle^{(\varphi)} \neq 0$. Suppose first $a$ has a prime order $p$ and consider the natural epimorphism of $\mathbb{Z}_{p}[x]$-modules mapping 1 to $a$ and $x$ to $\varphi(a)$ (regard $A$ as $\mathbb{Z}_{p}[x]$-module where $x$ acts as $\varphi$ ):

$$
F: \mathbb{Z}_{p}[x] \mapsto A .
$$

If $F$ is injective, we can replace $A$ by $\mathbb{Z}_{p}[x]$ and $\varphi$ by multiplication by $x$. If $H:=\mathbb{Z}_{p}\left[x^{2}\right]$, then $\varphi(H)=x H$ is infinite, while $H \cap x H=0$, a contradiction. Then, $F$ is not injective 
and $A$ is finite as it is isomorphic to a proper quotient of $\mathbb{Z}_{p}[x]$. If now $a$ has (any) order $p^{\epsilon}$, then $A / p A$ is finite, by the above. Moreover, $p A=\langle p a\rangle^{(\varphi)}$ is finite by induction on $\epsilon$.

(2) This can be proved in a similar way as case (3)

(3) We claim that if $a \in A$ has order $p^{\epsilon}$, then $\left|\langle a\rangle^{(\varphi)}\right| \leq p^{(m+1) \epsilon}$.

Assume first $\epsilon=1$, that is $a$ has order $p$ and $A_{0}:=\langle a\rangle^{(\varphi)}$ is elementary abelian. Suppose, by contradiction, that the above $F$ is injective. As above, let $H:=\mathbb{Z}_{p}\left[x^{2}\right]$. Then, $H_{(\varphi)}=\left(g\left(x^{2}\right)\right)$ for some polynomial $g$. Since $\left|H / H_{(\varphi)}\right|=p^{m}<\infty$, we have $g \neq 0$. Then $\left(g\left(x^{2}\right)\right) \nsubseteq H$, a contradiction. Therefore, for some $f \in \mathbb{Z}_{p}[x]$ with degree say $n$, we have

$$
\frac{\mathbb{Z}_{p}[x]}{(f)} \simeq_{\varphi}\langle a\rangle^{(\varphi)}=A_{0}
$$

Thus, the minimal $\varphi$-invariant subgroups of $A_{0}$ correspond $1-1$ to the irreducible monic factors of $f$, which are at most $n$. Consider a $\mathbb{Z}_{p}$-basis $X$ of A containing an element in each subgroup of them. The hyperplane $H$ of equation $x_{1}+x_{2}+\cdots+x_{n}=0$ has index $p$ in $\langle a\rangle^{(\varphi)}$ and $H_{(\varphi)}=0$ as $H \cap X=\emptyset$. Therefore, $\left|\langle a\rangle^{(\varphi)}\right| \leq p^{m+1}$.

If $\epsilon>1$, by induction $B:=\left\langle p^{\epsilon-1} a\right\rangle^{(\varphi)}$ has order at most $p^{(m+1)(\epsilon-1)}$ and $\langle a\rangle^{(\gamma)} / B$ has order at most $p^{m+1}$ by the case $\epsilon=1$. Therefore, $\left|a^{(\varphi)}\right| \leq p^{(m+1) \epsilon}$, as claimed.

In the general case, let $X$ be any subgroup of $A$ and $X_{(\varphi)}=0$. Thus, $|X|=: p^{\epsilon} \leq p^{m}$. Write $X=\left\langle a_{1}\right\rangle \oplus \cdots \oplus\left\langle a_{r}\right\rangle$ with $a_{i}$ of order $p^{\epsilon_{i}}$ and $\epsilon_{1}+\cdots+\epsilon_{r}=\epsilon$. Since $\left|\left\langle a_{i}\right\rangle^{(\varphi)}\right| \leq p^{(m+1) \epsilon_{i}}$ by the above claim, we have $\left|X^{(\varphi)}\right| \leq p^{(m+1) \epsilon}$. So that $\left|X^{(\varphi)} / X\right| \leq p^{(m+1) \epsilon-\epsilon} \leq p^{m^{2}}$.

Lemma 4.3 Let $D$ be a divisible periodic subgroup of an abelian group A. If $\varphi$ is either an inertial or LIN endomorphism of $A$, then $\varphi$ acts as multiplication on D.

Proof Without loss of generality, we may assume $D$ and $\varphi(D)$ are Prüfer groups. If $\varphi$ is LIN, then $D \leq \varphi(D)$ and thus $D=\varphi(D)$. Therefore, in both cases inertial or LIN, we have $\varphi(D) \leq D$.

We state next lemma without proof as it is a particular case of Theorem 1 of [4].

Lemma 4.4 Let $A$ be an elementary abelian p-group. If $\varphi$ is either inertial or LIN endomorphism of A, then $\varphi$ is FM (as in Proposition 3.4).

Lemma 4.5 If $A$ is an abelian p-group and $\varphi$ is either inertial or LIN endomorphism of $A$, then

(fs) $\quad \forall X \leq A\left|X^{(\varphi)} / X_{(\varphi)}\right|<\infty$.

In particular, if $\varphi$ is $L I N$, then $\varphi$ is inertial.

Proof We may assume $X_{(\varphi)}=0$. Thus, since $\varphi$ is multiplication on $D_{1}:=\operatorname{Div}(A)$, (see Lemma 4.3), we have $D_{1} \cap X=0$ and $X$ is reduced. Moreover, by Lemma 4.4, $\varphi$ is multiplication on a subgroup of finite index of $A[p]$ and we get that $X[p]$ is finite. It follows that $X$ is finite. Then (fs) holds by Lemma 4.2.

To shorten statements we introduce a definition.

Definition An abelian $p$-group $A$ is critical iff $D:=\operatorname{Div}(A) \neq 0$ has Min and $A / D$ is infinite but bounded.

Lemma 4.6 Let $A$ be an abelian p-group, $D:=\operatorname{Div}(A)$ and $\varphi$ an inertial endomorphism of $A$ 
(1) If $\varphi$ is not $F M$, then $A$ is critical.

(2) If $A=B \oplus D$ is critical, then $\varphi$ acts by multiplication on both $D$ and on a subgroup $B_{0}$ with finite index in $B$.

Proof First recall that (fs) holds by Lemma 4.5. We prove (1) by the following steps.

(I) A is not residually finite. Assume by contradiction it is. Note that if $\varphi$ is inertial, then there is no sequence of subgroups $X_{i}$ with the property that if we denote $Y_{i}:=X_{i} \cap \varphi\left(X_{i}\right)$ then we have:

(j) $Y_{i+1} \cap X_{i}=Y_{i}$

(jj) the sequence $\left|\varphi\left(X_{i}\right) / Y_{i}\right|$ is strictly increasing.

Otherwise, there would exists a subgroup $X_{\omega}:=\cup_{i} X_{i}$ with the properties that $\mid \varphi\left(X_{\omega}\right) / X_{\omega} \cap$ $\varphi\left(X_{\omega}\right)|\geq| \varphi\left(X_{i}\right) / Y_{i} \mid \geq i$ for each $i$.

On the other hand, we will construct a prohibited sequence $X_{i}$, getting the desidered contradiction. Let $X$ be any finite subgroup of $A$. By Lemma 4.2, the subgroup $K:=X^{(\varphi)}$ is finite. Since $A$ is residually finite, by (fs) there is a $\varphi$-subgroup $A_{*}$ with finite index in $A$ such that $A_{*} \cap K=0$. Now, as $\varphi$ is not multiplication on $\left(A_{*}+K\right) / K$, there is $a \in A_{*}$ such that $\varphi(a) \notin\langle a, K\rangle$. Let $Y:=X \cap \varphi(X), X^{\prime}:=\langle a\rangle+X$ and $Y^{\prime}:=X^{\prime} \cap \varphi\left(X^{\prime}\right)$. Let us check that

(j) $\varphi(X) \cap Y^{\prime}=Y$;

$\left(j j^{\prime}\right) \varphi\left(X^{\prime}\right)>\varphi(X)+Y^{\prime}$.

In fact, to prove $(j)$, if $\varphi(x) \in \varphi(X) \cap Y^{\prime}$ (where $x \in X$ ), then $\varphi(x)=m a+x_{0}$ with $m \in \mathbb{Z}, x_{0} \in X$ and $m a=\varphi(x)-x_{0} \in A_{*} \cap K=0$, hence $\varphi(x)=x_{0} \in Y$ and $(j)$ holds.

To prove $\left(j j^{\prime}\right)$, note that if $y^{\prime} \in Y^{\prime}=X^{\prime} \cap \varphi\left(X^{\prime}\right)$, then $\exists n, m \in \mathbb{Z}, \exists x, x_{0} \in X$ such that $y^{\prime}=m a+x=n \varphi(a)+\varphi\left(x_{0}\right)$. Then, $m a-n \varphi(a) \in A^{*} \cap K=0$. Hence $x=\varphi\left(x_{0}\right) \in$ $Y:=X \cap \varphi(X)$. Since $\varphi(a) \notin\langle a, K\rangle$, then $p$ divides $s$, and so $Y^{\prime} \leq\langle p \varphi(a)\rangle+Y \not \supset \varphi(a)$. Therefore, $\left(j j^{\prime}\right)$ holds as $\varphi(a) \in \varphi\left(X^{\prime}\right) \backslash\langle p \varphi(a)\rangle+Y$.

Thus, we can define by induction a prohibited sequence as the above one, since from $(j)$ and $\left(j j^{\prime}\right)$ it follows $\left|\varphi\left(X^{\prime}\right) / Y^{\prime}\right|>|\varphi(X) / Y|$ and we get a contradiction.

(II) $A$ is not reduced. Otherwise, let $R$ be a basic subgroup of $A$. By (fs), $R^{(\varphi)} / R$ is finite and so $H:=R^{(\varphi)}$ is residually finite as well. Also, $A / H$ is divisible. By step (I) applied to $H$, there are a $p$-adic $\alpha$ and a finite $\varphi$-invariant subgroup $A_{1}$ of $H$ such that $\varphi=\alpha$ on $H / A_{1}$. As the kernel $K / A_{1}$ of $(\varphi-\alpha)_{\mid A / A_{1}}$ contains $H / A_{1}$ and its image is reduced, while $A / H$ is divisible, it is clear that $K=A$ and $\varphi=\alpha$ on $A / A_{1}$, the desidered contradiction.

(III) $A$ is critical. Let $A=B \oplus D$ with $B$ infinite but reduced. As (fs) holds, at the expense of substituting a finite index $\varphi$-invariant subgroup $A_{0}$ for $A$, we may assume that $B$ is $\varphi$-invariant. Further, by step (II) and Lemma 4.3, $\varphi$ is multiplication on both $D$ and on a finite index subgroup of $B$. So we may also assume $\varphi$ is multiplication on $B$. Let $\varphi$ act on $B$ and $D$ by means of $p$-adics $\alpha_{1}, \alpha_{2}$, respectively, As $\varphi$ is not FM, we have $\alpha_{1}$ and $\alpha_{2}$ act differently on $B$.

If by contradiction $B$ is unbounded, then there is a quotient $B / S \simeq \mathbb{Z}\left(p^{\infty}\right)$. By (fs) we can assume $S$ to be $\varphi$-invariant and consider $\bar{A}:=A / S$. This a divisible group on which $\varphi$ acts as a (universal) multiplication by Lemma 4.3, contradicting the assumption on $\alpha_{1}$ and $\alpha_{2}$. So that $B$ is bounded.

If by contradiction $D$ has infinite rank, we may substitute $B\left[p^{\epsilon}\right]$ for $B$ where $\epsilon$ is the smallest natural number such that $B / B\left[p^{\epsilon}\right]$ is finite. By the reduced case above, $\varphi$ is multiplication on a subgroup $A_{*}$ of finite index of $A\left[p^{\epsilon}\right]$. Then, as $D$ has infinite rank, $\alpha_{1} \equiv \alpha_{2}$ mod $p^{\epsilon}$ and $\varphi$ is multiplication on $\left(B \cap A_{*}\right) \oplus D$ which has finite index in $A$, a contradiction. 
To prove (2) recall that $\varphi$ acts as multiplication on $D$ by Lemma 4.3. The other part follows from (fs) and part (1) of this Lemma.

Proof of Proposition 2.2 We start by proving necessity in part (R).

(1) Let us show that there are subgroups $A_{0}, B, C, D$ as in the statement and (i), (ii) and (iii) hold. By Proposition 4.1, we reduce trivially to the case when $A$ is a p-group. Then, note that if all $\varphi_{i}$ 's are FM, then clearly there is a finite index subgroup $C$ of $A$ such that all $\varphi_{i}$ 's acts as multiplication on $C$. Otherwise, by Lemma 4.6, $A=B_{0} \oplus D$ is critical, with $B_{0}$ bounded and $D$ divisible with finite rank. For each $i$, there is a finite index $\varphi_{i}$-invariant subgroup $B_{i}$ of $B_{0}$ such that $\varphi_{i}$ acts as multiplication on $B_{i}$. Let then $B:=\cap_{i} B_{i}$. Then, $A_{0}:=B+D$ is the desired subgroup as in the statement.

(2) if (i), (ii) and (iii) hold, then(FS) holds. Note that as $A / A_{0}$ and $\varpi(B \oplus D)$ are finite, then $C$ contains all but finitely many primary components of $A$. Therefore, we reduce again to the case when $A$ is a p-group and either $A_{0}=C$ or $A_{0}=B \oplus D$ is critical.

(2.1) In the former subcase, each $\varphi_{i}$ is FM, that is it acts as multiplication (by the $p$-adic $\alpha_{i}$ ) on $A_{0}$ (which has finite index in $A$ ) and modulo a subgroup with finite order $F_{i}:=\operatorname{im}\left(\varphi_{i}-\alpha_{i}\right)$, see Proposition 3.4. Then, all $\varphi_{i}$ are multiplication $A_{0}$ and modulo the finite subgroup $F_{0}:=F_{1} \oplus \cdots \oplus F_{l}$. Therefore, for each $X \leq A$, we have that $X \cap A_{0}$ and $X+F$ are $\varphi_{i}$-invariant for each $i$.

(2.2) In the latter subcase, $A_{0}=B \oplus D$ and $\varphi_{i}$ is multiplication on $B$ and $D$, where $B$ is bounded and $D$ divisible with finite rank. Let $X_{0}:=X \cap A_{0}$. Then, $\left|X / X_{0}\right| \leq$ $\left|A / A_{0}\right|$ is finite. Further, $X_{*}:=(D \cap X)+(B \cap X)$ is $\varphi_{i}$-invariant and the group $X_{0} / X_{*}$ is bounded as $B$ is. Also $X_{0} / X_{*}$ has rank $r$ at most the rank of $D$, hence $X_{0} / X_{*}$ is finite. Thus, $X / X_{\Phi}$ is finite. Hence, each $\varphi_{i}$ is LIN on $A$. By Lemma $4.5, \varphi$ is even inertial. To show that $X^{\Phi} / X$ is finite as well, note that there is $m$ such that $X / X_{\Phi}$ is contained in the $m$ th socle $\left(A / X_{\Phi}\right)[m]$ on which each $\varphi_{i}$ is FM by Lemma 4.6. Thus, $X^{\Phi} / X$ is finite as in (2.1) above.

To prove sufficiency in part $(R)$, note that $(F S)$ implies trivially that each $\varphi_{i}$ is inertial.

To prove necessity in part $(L)$, let all $\varphi_{i}$ 's be LIN. Assume first $A=A_{p}$ is a $p$-group. Then, by Lemma 4.5, all $\varphi_{i}$ 's have (fs) and therefore are inertial. Thus, by the above, there are subgroups $A_{0}, B, D, C$ as in part (R) of the statement and ( $i$ ), (ii), ( $\left.i i i\right)$ hold. If $B \neq 0$ (hence $C=0$ ) we can assume $B$ is infinite and, by Proposition 3.1, each $\varphi_{i}$ is invertible on $B$ and $\varphi_{i} \neq 0$ on $D$ (if $D \neq 0$ ). On the other hand, if some $\varphi_{i}$ is not invertible on $C \neq 0$ (hence $B \oplus D=0$ ), then $C$ has Min and we can put $A_{0}:=\operatorname{Div}(C)$ and the statement holds. Thus, we have proved that the condition is necessary for the $p$-components of $A$.

In the general case, apply Proposition 4.1 and deduce that again LIN implies inertial since this is true on the $p$-components (Lemma 4.5). Moreover, the set $\pi$ of primes such that some $\varphi_{i}$ is not an invertible multiplication on $A_{p}$ is finite, by Proposition 4.1 again. By the above, for each $p \in \pi$, there is a finite index subgroup $A_{0, p}$ of $A_{p}$ such that $A_{0, p},=B_{p} \oplus D_{p} \oplus C_{p}$ as in part (R) of the statement and (i), (ii), (iii) hold. Then, the statement (in the general case) holds with:

$$
A_{0}=A_{\pi^{\prime}} \oplus \bigoplus_{p \in \pi} A_{p}, \quad B:=\bigoplus_{p \in \pi} B_{p}, \quad D:=\bigoplus_{p \in \pi} D_{p} \text { and } C:=A_{\pi^{\prime}} \oplus \bigoplus_{p \in \pi} C_{p} .
$$

To prove sufficiency in part $(L)$ note that, arguing componentwise, by Propositions 2.1 and 4.1, it is enough to show that each $\varphi_{i}$ is LIN on $B \oplus D$ as in the statement and in the case when this is a $p$-group. For each $X \leq B \oplus D$ let $X_{*}:=(X \cap B)+(X \cap D)$. Then, $X / X_{*}$ is finite being bounded and of finite rank. Moreover, since clearly $\varphi(X \cap B)=X \cap B$ and $\varphi(X \cap D)$ has finite index in $X \cap D$, we have $X_{*} / X_{*} \cap \varphi\left(X_{*}\right)$ is finite as well. 


\section{Inertial endomorphisms of a non-periodic abelian group}

This section is devoted to the proof of Proposition 2.3. In next statement, we recall some well-known facts.

Lemma 5.1 Let $A_{1}$ be a subgroup of an abelian group $A$ and $\pi$ a set of primes.

(1) If $A / A_{1}$ is a $\pi^{\prime}$-group, then $A$ is $\pi$-divisible iff $A_{1}$ is $\pi$-divisible.

(2) If $A$ if torsion free, $A / A_{1}$ periodic and $A_{1}$ is $\pi$-divisible then $A / A_{1}$ is a $\pi^{\prime}$-group and $A$ is $\pi$-divisible.

(3) If $A_{1}$ is torsion free and $\pi$-divisible while $A / A_{1}$ is $\pi^{\prime}$-group, then multiplication by a $\pi$-number is invertible.

Next, Lemma is a generalization of Lemma 4.2.(1) to non-periodic groups.

Lemma 5.2 Let $A$ be an abelian group, $a \in A$ and $\varphi \in E(A)$. If $\varphi$ is either inertial or LIN, then the torsion subgroup $T$ of the $\varphi$-submodule generated by a is finite.

Proof We may assume $A=\langle a\rangle^{(\varphi)}$. If $a$ has finite order, apply Lemma 4.2.(1). Assume $a$ is aperiodic. By Proposition 3.3, $\varphi=\frac{m}{n}$ on $A / T$ ( $m, n$ coprime), that is $(n \varphi-m)(a)$ is periodic. Regard $A$ as $\mathbb{Z}[x]$-module (where $x$ acts as $\varphi$ ) and consider the natural epimorphism mapping 1 to $a$ and $x$ to $\varphi(a)$ :

$$
F: \mathbb{Z}[x] \mapsto A .
$$

Let $I$ be the inverse image of $T$ via $F$. Then, $(n x-m) \subseteq I$ and $\mathbb{Z}[x] / I \simeq A / T$ is torsion free (as $\mathbb{Z}$-module). Since proper quotients of $\mathbb{Z}[x] /(n x-m) \simeq \mathbb{Z}[1 / n]=\mathbb{Q}^{\varpi(n)}$ are periodic, then $I=(n x-m)$. Applying $F$ we get that $T=\mathbb{Z}[\varphi]\langle(m \varphi-n)(a)\rangle$ is a cyclic $\varphi$-submodule. It is finite by Lemma 4.2.

Proof of Proposition 2.3,necessity Assume that $\varphi$ is either inertial or LIN. By Proposition 3.3, $\varphi$ is multiplication by $\frac{m}{n} \in \mathbb{Q}$ on $A / T$, where $T:=T(A)$ and $m, n$ are coprime. Thus, $\varphi=\frac{m}{n}$ on each $\varphi$-invariant torsion-free section of $A$. Let $\pi:=\varpi(n)$. We proceed by a sequence of claims.

(1) There is a free abelian $F \leq A$ such that $V:=\mathbb{Z}[\varphi] F$ is torsion free and $A / V$ is periodic. In fact, by Zorn's Lemma, there is a subset $S$ of $A$ which is maximal with respect to " $F:=\langle S\rangle$ is free abelian on $S$ and $V:=\mathbb{Z}[\varphi]\langle S\rangle$ is torsion free". It follows that $A / V$ is periodic. If not, there is an aperiodic $a \in A$ such that $\langle a\rangle \cap V=0$. By Lemma 5.2, the torsion subgroup of $\mathbb{Z}[\varphi]\langle a\rangle$ has finite order $s$. Thus, $\mathbb{Z}[\varphi]\left\langle a^{s}\right\rangle \simeq \mathbb{Q}^{\varpi(n)}$ has rank 1 (see Proposition 3.3) and $\left\{a^{S}\right\} \cup S$ has the above properties instead of $S$, a contradiction. Then $V$ is torsion free subgroup and $A / V$ is periodic.

(2) the $\pi$-component $A_{\pi}$ is bounded. To establish this, assume by contradiction that $T$ has a quotient $T / K$ isomorphic to a Prüfer $p$-group, with $p \in \pi$. By (FS) of Proposition $2.2, K^{(\varphi)} / K$ is finite. Thus, without loss of generality, we may assume $K^{(\varphi)}=0$, that is $T$ is a Prüfer $p$-group. Since $V$ contains a $\varphi$-invariant subgroup isomorphic to $\mathbb{Q}^{\pi}$, then $T+V$ contains a $\varphi$-invariant subgroup isomorphic to $\mathbb{Z}\left(p^{\infty}\right) \oplus \mathbb{Q}^{\pi}$ and it is enough to check that:

- the endomorphism $\varphi=\alpha \oplus \frac{m}{n}$ of $\mathbb{Z}\left(p^{\infty}\right) \oplus \mathbb{Q}^{\pi}$ is neither inertial nor LIN ( $\alpha$ any $p$-adic). To this aim consider the "diagonal" subgroup

$$
H=\left\{\left[t p^{-i}\right] \oplus t p^{-i} \mid i \in \mathbb{N}, t \in \mathbb{Z},\left[t p^{-i}\right] \in \mathbb{Q}^{\{p\}} / \mathbb{Z}\right\}
$$


and note $(m-n \varphi)\left(\left[p^{-i}\right] \oplus p^{-i}\right)=(m-n \alpha)\left[p^{-i}\right] \oplus 0$. Since $p$ does not divide $m$, we have $\mathbb{Z}\left(p^{\infty}\right)=(m-n \varphi)(H)$. Therefore both $H$ and $\varphi(H)$ have infinite index in $H+\varphi(H)$, as desired.

(3) If $r_{0}(A)<\infty$, then (b) holds. This is now clear.

(4) If $r_{0}(A)=\infty$ and $\varphi$ is inertial, then (a) holds. By Proposition 3.3, we know that $\varphi=m \in \mathbb{Z}$ on $V=F$, which is a free abelian group as in claim (1). So there exists a ( $\varphi$-invariant) subgroup $W$ such that $V / W$ is a periodic group whose $p$-component is divisible with infinite rank for each prime $p$. By the periodic case, Proposition 2.2, $\varphi$ is FM on $A / W$, since it contains a divisible $p$-group with infinite rank for each prime $p$. Without loss of generality, we can assume $\varphi$ is a multiplication indeed on $A / W$. Moreover, since each $p$-component of $V / W$ is unbounded, we have $\varphi=m$ on $A / W$. Then $W \geq \operatorname{im}(\varphi-m) \simeq A / \operatorname{ker}(\varphi-m)$ where the former is torsion free and the latter is periodic, as a factor of $A / V$. Thus $\varphi=m$ on $A$.

The proof of necessity in the case when $\varphi$ is inertial is now complete. Let us consider the case when $\varphi$ is LIN.

(5) If $r_{0}(A)=\infty$ and $\varphi$ is LIN, then (a) holds. Let $F$ and $V=\mathbb{Z}[\varphi] F$ as in claim (1) above. By Proposition 3.3, $m=1$, that is $\varphi=\frac{1}{n}$ on $V$. Then $V / F$ is the sum of infinitely many copies of $\mathbb{Z}\left(p^{\infty}\right)$ for each prime $p \in \pi:=\varpi(n)$. Take $F_{*}$ such that $F / F_{*}$ is a periodic $\pi^{\prime}$-group whose $p$-component is divisible with infinite rank for each prime $p \in \pi^{\prime}$. Let $V_{*} / F_{*}$ be the $\pi$-component of $V / F_{*}$. Since $V / V_{*}$ is a $\pi^{\prime}$-group, $V_{*}$ is $\pi$-divisible, by Lemma 5.1.(1). Thus $V_{*}$ is $\varphi$-invariant and $V / V_{*} \simeq{ }_{\varphi} F / F_{*}$. Let $A_{*} / V_{*}$ be the $\pi^{\prime}$-component of $A / V_{*}$.

We claim $A / A_{*}$ is finite. It is enough to check that the $\pi$-component $A_{1} / V_{*}$ of $A / V_{*}$ is finite. To this aim, notice that $T_{1}:=T\left(A_{1}\right)=A_{\pi}$. On one hand $A_{1} /\left(T_{1}+V_{*}\right)$ is a $\pi$-group by definition of $A_{1} / V_{*}$; on the other hand $A_{1} /\left(T_{1}+V_{*}\right)$ is $\pi^{\prime}$-group as $\left(T_{1}+V_{*}\right) / T_{1}$ is $\pi$-divisible and $A_{1} / T_{1}$ is torsion free [see Lemma 5.1.(2)]. Thus $A_{1}=T_{1} \oplus V_{*}$ and the claim reduces to show $T_{1}$ is finite. Since by (2) above, $T_{1}=A_{\pi}$ is bounded, we assume by contradiction that $T_{1}$ has infinite rank. By Proposition 2.2, we have that $\varphi$ is FM on $T_{1}$. Then, there exists a prime $p \in \pi$ such that $\varphi=s \in \mathbb{Z}$ is a multiplication by $s$ not multiple of $p$ on a countable $\mathbb{Z}_{p}$-submodule $B=\oplus_{i}\left\langle b_{i}\right\rangle$ of $T_{1}$. Let $\left\{a_{i} \mid i<\omega\right\}$ be a countable subset of the above basis $S$ for $F$ and set $W:=\left\langle V_{i} \mid i<\omega\right\rangle=\oplus_{i} V_{i}$, where $V_{i}:=\mathbb{Z}[\varphi] a_{i}$. Also, let $M:=B \oplus W$ and $H:=\left\langle a_{i}+b_{i} \mid i<\omega\right\rangle$ its "diagonal" subgroup, which is free on the $\mathbb{Z}$-basis of the $a_{i}+b_{i}$ 's. Since $\varphi$ is one-to-one on $M$, then $\varphi(H)$ is torsion free as $H$ is. Recall that $\varphi=\frac{1}{n} \in \mathbb{Q}$ on $V$. Then for all $i$ we have: $H+\varphi(H) \ni(p-n \varphi)\left(a_{i}+b_{i}\right)=(p-1) a_{i}$, as $p$ divides $n$. Since $p a_{i} \in H$, then $a_{i} \in H+\varphi(H)$. Thus $B \leq H+\varphi(H)$. Therefore $(H+\varphi(H)) / \varphi(H) \geq$ $(B+\varphi(H)) / \varphi(H) \simeq B$ is infinite, contradicting $\varphi$ is LIN. Thus $A / A_{*}$ is finite.

Let us show that $\varphi=\frac{1}{n}$ on some $A_{0}$ with finite index in $A_{*}$. Recall that $A_{*} / V_{*}$ is a $\pi^{\prime}$-group and its $p$-component contains a divisible $p$-group of infinite rank (for each prime $\left.p \in \pi^{\prime}\right)$. Then, by Proposition 2.2, we have that $\varphi$ is FM on $A_{*} / V_{*}$. Thus, $\varphi$ is multiplication on some $A_{0} / V_{*}$ with finite index in $A_{*} / V_{*}$. On the one hand, $\varphi=\frac{1}{n}$ on $V$. On the other hand, by Lemma 5.1.(3) the multiplication by $\frac{1}{n}$ is an endomorphism on the whole $A_{0}$. Then, as $\operatorname{ker}\left(\varphi_{\mid A_{0}}-\frac{1}{n}\right) \leq V$ and $\left(\varphi-\frac{1}{n}\right)\left(A_{0}\right) \leq V_{*}$, we have that $A_{0} / \operatorname{ker}\left(\varphi_{\mid A_{0}}-\frac{1}{n}\right) \simeq\left(\varphi-\frac{1}{n}\right)\left(A_{0}\right)$ is both periodic and torsion free. Therefore, $\varphi=\frac{1}{n}$ on $A_{0}$. Thus, $(a)$ holds.

Proof of Proposition 2.3, sufficiency We treat both conditions inertial and LIN simultaneously.

If $\varphi$ is as in (a), it is trivial that $\varphi$ is inertial (or LIN, respectively). Let then $\varphi$ be as in $(b)$. We have to show that for each subgroup $X$ of $A$ the statement $R(X)$ [respectively, $L(X)$ ] below holds. 


$$
R(X):=\left(\left|\frac{X+\varphi(X)}{X}\right|<\infty\right) \quad L(X):=\left(\left|\frac{X+\varphi(X)}{\varphi(X)}\right|<\infty\right)
$$

Let $\pi:=\varpi(n)$. We proceed by a sequence of claims.

(6) $\varphi=\frac{m}{n}$ is inertial on $A / T$ which is $\pi$-divisible.

In fact, if $a \in A$, there is a nonzero integer s such that $s a \in V$. Thus $s(n \varphi-m)(a)=$ $(n \varphi-m)(s a)=0$ and $(n \varphi-m)(A) \subseteq T$, as claimed.

(7) If $X$ is any periodic subgroup, then $R(X)$ [respectively, $L(X)$ ] holds.

This follows straightforward, since $X^{(\varphi)} \cap V=0$ and one can verify $R(X)$ [respectively, $L(X)] \bmod V$.

(8) If for each torsion-free subgroup $Y / A_{\pi} \leq A / A_{\pi}$ it holds $R\left(Y / A_{\pi}\right)$ [respectively, $L\left(Y / A_{\pi}\right)$ ], then for each torsion-free subgroup $X \leq A$ it holds $R(X)$ [respectively, $L(X)]$.

Recall that by hypothesis $B:=A_{\pi}$ is bounded by some $e$. Clearly, $X$ has finite rank. Let $\left|\frac{X+\varphi(X)+B}{X+B}\right|=: s<\infty$. Then, $s \varphi(X) \leq X+B$. Thus, $\operatorname{es} \varphi(X) \leq X$. Since $\varphi(X)+X / X$ is bounded and has finite rank, it is finite. Then, $R(X)$ holds, as wished. Similarly, if $\left|\frac{X+\varphi(X)+B}{\varphi(X)+B}\right|=s<\infty$, then $s X \leq \varphi(X)+B$, hence es $X \leq \varphi(X)$ and $(\varphi(X)+X) / \varphi(X)$ is finite.

(9) If $A_{\pi}=0$ and $X$ is torsion free then $R(X)$ [respectively, $\left.L(X)\right]$ holds.

As the hypotheses on $\varphi$ hold even in $A_{1}:=\mathbb{Z}[\varphi] X$ with respect to $V_{1}:=A_{1} \cap V$, we can assume $A=A_{1}$, that is $X$ has maximal torsion-free rank $r$ and $V \simeq \mathbb{Q}^{\pi} \oplus \ldots \oplus \mathbb{Q}^{\pi}$ ( $r$ times).

Let $K / X$ be the $\pi$-component of $A / X$ (which is periodic). By hypothesis $R(K+V)$ holds, thus $R(K)$ holds, since $(K+V) / K \simeq V /(V \cap K)$ is finite as it is a $\pi^{\prime}$-group. On the other hand, $K$ is torsion free, as $T$ is a $\pi^{\prime}$-group. Thus, $T(K+\varphi(K))$ is finite. Let $Y:=X+\varphi(X), Y_{R}:=Y \cap(X+T)$ and $Y_{L}:=Y \cap(\varphi(X)+T)$. On the one hand, $Y_{R} / X$ and $Y_{L} / \varphi(X)$ are both finite, as isomorphic to quotients of $Y \cap T \leq$ $T(K+\varphi(K))$, which is finite. On the other hand, by (6), we have $R(X+T)$ [respectively, $L(X+T)]$. Therefore, $\left|Y / Y_{R}\right|=|(Y+T) /(X+T)|<\infty$ (respectively, $\left|Y / Y_{L}\right|=$ $|(Y+T) /(\varphi(X)+T)|<\infty)$. Thus $\mathrm{R}(\mathrm{X})$ [respectively, $\mathrm{L}(\mathrm{X})]$ holds.

Thus, we are reduced to show the following, which completes the proof.

(10) If $R\left(X_{0}\right)$ [respectively, $L\left(X_{0}\right)$ ] holds for each torsion-free subgroup $X_{0}$ of $A$, then $R(X)$ [respectively, $L(X)$ ] holds for any subgroup $X$.

By (7) above, $\varphi$ induces on $T$ a inertial (resp LIN) endomorphism. Let $U:=T(X)$. By Proposition 2.2 applied to $T$, we have (FS), so that $U / U_{(\varphi)}$ is finite. Since the hypotheses hold modulo $U_{(\varphi)}$ (which is periodic), that is for the endomorphism induced by $\varphi$ on the group $A / U_{(\varphi)}$, we can assume $U_{(\varphi)}=0$ that is $U=T(X)$ is finite. Therefore, $X=X_{0} \oplus U$ splits on $U$. Since $X / X_{0}$ is finite, then $R\left(X_{0}\right)$ [respectively, $L\left(X_{0}\right)$ ] implies straightforward $R(X)$ [respectively, $L(X)]$.

\section{Proofs of Theorem $A$ and remaining results}

We state a lemma dealing with finitely many inertial endomorphisms. Denote by $\oplus_{r} \mathbb{Q}^{\pi}$ the direct sum of $r$ copies of $\mathbb{Q}^{\pi}$.

Lemma 6.1 Let $\varphi_{1}, \ldots, \varphi_{t}$ be finitely many inertial endomorphisms of an abelian group $A$ with $r:=r_{0}(A)<\infty$. 
If $\varphi_{i}=\frac{m_{i}}{n_{i}} \in \mathbb{Q}$ on $A / T$, then there is a $\mathbb{Z}\left[\varphi_{1}, \ldots, \varphi_{t}\right]$-submodule $V \simeq \oplus_{r} \mathbb{Q}^{\pi}$, where $\pi:=\varpi\left(n_{1} \cdots n_{l}\right)$, such that $A / V$ is periodic.

Proof It is easily seen that for each $p_{j} \in \pi$ there is $\psi_{j} \in \mathbb{Z}\left[\varphi_{1}, \ldots, \varphi_{t}\right]$ such that $\psi_{j}=r_{j} / p_{j}$ on $A / T$ ( $r_{j}$ and $p_{j}$ coprime). Then, there are coprime $m, n$ such that $\varphi:=\sum_{j} \psi_{j}=m / n$ on $A / T$ where $\varpi(n)=\pi$.

By Proposition 2.3, for each $i$, there is a torsion free $\varphi_{i}$-invariant subgroup $V_{i}$ such that $A / V_{i}$ is periodic. Pick $\mathbb{Z}$-independent elements $b_{1}, \ldots, b_{r}$ of $\cap_{i} V_{i}$, where $r=r_{0}(A)$. By Lemma 5.2, for each $k$, there exists $a_{k} \in\left\langle b_{k}\right\rangle$ such that $\mathbb{Z}[\varphi]\left\langle a_{k}\right\rangle$ is torsion free with rank 1 . Clearly, $V \simeq \oplus_{r} \mathbb{Q}^{\pi}$. As $V$ has maximal torsion-free rank, it is plain that $A / V$ is periodic.

We claim $\varphi_{i}(V) \subseteq V$. Set $W_{i}:=\mathbb{Z}\left[\varphi_{i}\right]\left\langle a_{1}, \ldots, a_{r}\right\rangle \leq V_{i}$. Since $\varphi_{i}=\frac{m_{i}}{n_{i}}$ on $W_{i}, \varphi=\frac{m}{n}$ on $V$ and $\varpi\left(n_{i}\right) \subseteq \varpi(n)$, we have $V_{i} \leq V$. Also $V / W_{i}$ is a $\pi$-group, as $V /\left\langle a_{1}, \ldots, a_{r}\right\rangle$ is such. Let $a$ be any element of $V$ and $e$ be the bound of $A_{\pi}$ (which is bounded by Proposition 2.3). Therefore, there is a $\pi$-number $t$ such that $t a \in W_{i}$ hence $\left(n_{i} \varphi_{i}-m_{i}\right)(t a) \in T \cap W_{i}=0$. Thus $\left(n_{i} \varphi_{i}-m_{i}\right)(a) \in A_{\pi}$, so that $e\left(n_{i} \varphi_{i}-m_{i}\right)(a)=0$. Since $e$ and $n_{i}$ are $\pi$-numbers and $V$ is $\pi$-divisible, we have $\varphi_{i}(V)=e n_{i} \varphi_{i}(V)=e m_{i} V \subseteq V$ as claimed.

Proof of Theorem A, necessity Assume all $\varphi_{i}$ are inertial. If $r_{0}(A)=\infty$, then by Proposition 2.3 for each $i$ there is a $\varphi_{i}$-invariant subgroup $A_{i}$ with finite index such that each subgroup of $A_{i}$ is $\varphi$-invariant. Then, $(a)$ holds with $A_{0}:=\cap_{i} A_{i}$.

Assume now $r:=r_{0}(A)<\infty$. By Lemma 6.1 , there is a $\mathbb{Z}\left[\varphi_{1}, \ldots, \varphi_{t}\right]$-submodule $V$ such that $V \simeq \oplus_{r} \mathbb{Q}^{\pi}$ and $A / V$ is periodic. Let $\pi_{2}$ be the set of primes $p$ such that some $\varphi_{i}$ is not FM on the $p$-component of $A / V$. Note that the definition of $\pi_{2}$ is independent of $V$, as all possible $V$ are commensurable each other.

On the one hand from Proposition 2.2, it follows that $\pi_{2}$ is finite and for each $p \in \pi_{2}$ the $p$-component $A_{p}$ of $A$ is the sum of a bounded subgroup and a finite rank divisible subgroup. On the other hand, $A_{\pi}$ is bounded, by Proposition 2.3. Thus, if $\pi_{1}:=\pi \cup \pi_{2}$, there is $C^{*}$ such that

$$
A=A_{\pi_{1}} \oplus C^{*} .
$$

By Proposition 2.2 and the definition of $\pi_{1}$, there is a finite index subgroup $B \oplus D$ of $A_{\pi_{1}}$ such that $B$ is bounded, $D$ is divisible with finite rank hence a $\pi^{\prime}$-group and each $\varphi_{i}$ acts as multiplications on both $B$ and $D$, as we claim in the statement. Let us identify a suitable $C$.

We may assume $V \leq C^{*}$. In fact $\left|V /\left(V \cap C^{*}\right)\right|=: s$ is finite as $V \simeq \oplus_{r} \mathbb{Q}^{\pi}$ and $V /\left(V \cap C^{*}\right) \simeq\left(V+C^{*}\right) / C^{*}$ is periodic with bounded $\pi$-component. So we may substitute $s V$ for $V$ and get $V \leq C^{*}$.

Use bar notation in $\bar{A}:=A / V$. Consider the primary decomposition $\bar{C}^{*}=\bar{C}_{1}^{*} \oplus \bar{C}_{0}^{*}$ where $\bar{C}_{1}^{*}$ (respectively, $\bar{C}_{0}^{*}$ ) is a $\pi_{1}$-group (respectively, $\pi_{1}^{\prime}$-group). By (FS) of Proposition 2.2 and the definition of $\pi_{1}$, each $\varphi_{i}$ is multiplication on a subgroup $\bar{C}_{0}$ with finite index in $\bar{C}_{0}^{*}$. On the other hand, $C_{1}^{*}$ is torsion free (with finite rank) hence $\bar{C}_{1}^{*}$ has Min. Thus, $\bar{C}_{1}^{*}$ has a divisible finite index subgroup, say $\bar{C}_{1}$, on which each $\varphi_{i}$ is multiplication (see Lemma 4.3). Therefore, the subgroup $C:=C_{1}+C_{0}$ has finite index in $C^{*}$ and each $\varphi_{i}$ is multiplication on $\bar{C}$. So conditions (i), (ii), (iii) of the statement hold for $A_{0}:=B \oplus D \oplus C$.

To prove condition (iv), for each $i$ let $\varphi_{i}=\frac{m_{i}}{n_{i}} \in \mathbb{Q}$ on $V$ (see Proposition 3.3) and $p \in \pi(D)$ such that the $p$-component $\bar{C}_{p}$ of $\bar{C}$ is infinite (hence unbounded). On the one hand, as $C_{p}$ is torsion free, we get that $\varphi_{i}=\frac{m_{i}}{n_{i}}$ on $C_{p}$. On the other hand, $\varphi_{i}$ acts by the same $p$-adic $\alpha$ on $D_{p}$ as on $\bar{D}_{p}$. Therefore $\alpha=\frac{m_{i}}{n_{i}}$ by Proposition 2.2 (as $\bar{D}_{p} \oplus \bar{C}_{p}$ is non-critical). 
Proof of Theorem A, sufficiency It is clear that if $(a)$ holds, then all $\varphi_{i}$ are inertial, so only case $(b)$ is left. By Proposition 2.1, we may assume $A=A_{0}$. Fix $a_{1}, \ldots, a_{r}$ such that $V:=\mathbb{Z}\left[\varphi_{1}, \ldots, \varphi_{t}\right]\left\langle a_{1}, \ldots a_{r}\right\rangle$. Fix $i$ and let $V_{i}:=\mathbb{Z}\left[\varphi_{i}\right]\left\langle a_{1}, \ldots a_{r}\right\rangle$. Then $V / V_{i}$ is a divisible $\pi$-group with finite rank. By Proposition 2.3, $\varphi_{i}$ is inertial iff it is such on the periodic group $A / V_{i}$. Clearly, by Proposition $2.2, \varphi_{i}$ is already inertial on $A / V$. Use bar notation in $\bar{A}:=A / V_{i}$.

If $p \in \pi$ (which is a finite set), the $p$-component of $\bar{A}$ is $\bar{A}_{p} \oplus \overline{C_{(p)}}$, where $A_{p}$ is the $p$-component of $A$ and $\overline{C_{(p)}}$ is the $p$-component of $\bar{C}$. Clearly, $\overline{C_{(p)}}$ contains $\overline{V_{(p)}}$, the $p$ component of $\bar{V}$. Since $C$ has no elements of order $p$, then $C_{(p)}$ is torsion free and, by Lemma 5.1, $\overline{C_{(p)}} / \overline{V_{(p)}}$ is a $\pi^{\prime}$-group. Hence $\overline{C_{(p)}}=\overline{V_{(p)}}$. Therefore, $\varphi_{i}$ is multiplication on $\overline{C_{(p)}}$. Moreover, this subgroup is divisible of finite rank. On the other hand, $\varphi_{i}$ is multiplication even on $\bar{A}_{p} \simeq_{\varphi_{i}} A_{p} \leq B$, which is bounded. Thus, we are in a position to apply Proposition 2.2 and obtain that $\varphi_{i}$ is inertial on $\bar{A}_{p} \oplus \overline{C_{(p)}}$, the $p$-component of $\bar{A}$.

If $p \notin \pi$, then the $p$-component of $\bar{A}$ is $\varphi_{i}$-isomorphic to the $p$-component of $A / V$ as $V / V_{i}$ is a $\pi$-group.

We have seen that $\varphi_{i}$ is inertial on all p-components of $\bar{A}$ and even multiplication on all but finitely many, thus $\varphi$ is inertial on the whole of $A$ by Proposition 4.1.

Proof of Corollary A Apply Theorem A to any pair of inertial endomorphisms $\varphi_{1}, \varphi_{2}$ of an abelian group $A$. If $(a)$ holds for both $\varphi_{1}$ and $\varphi_{2}$, then $\varphi_{1} \varphi_{2}-\varphi_{2} \varphi_{1}=0$ on a subgroup with finite index of $A$, since multiplications commute. Otherwise, by Proposition 3.3, $\varphi_{1}$ and $\varphi_{2}$ commute on $A / T$ anyway, where $T=T(A)$. Moreover, there is a subgroup $A_{0}$ with finite index of $A$ such that $\varphi_{1}$ and $\varphi_{2}$ commute on $A_{0} / V$ for some $V$ as in Theorem A. As $T \cap V=0$, then $\varphi_{1}$ and $\varphi_{2}$ commute on $A_{0}$, as wished.

Proof of Theorem $B$ It is enough to prove the statement for a finitely generated subgroup $\Gamma=\left\langle\varphi_{1}, \ldots, \varphi_{t}\right\rangle$ of $\operatorname{IAut}(A)$. If case $(a)$ of Theorem A applies statements (1) and (2) follow trivially. Otherwise, let $A_{0}$ and $V$ be as in case $(b)$ of Theorem A and $T_{0}:=T\left(A_{0}\right)$. Then, $\Gamma^{\prime}$ acts trivially on both $A_{0} / V$ and $A_{0} / T_{0}$. Thus, $\Gamma^{\prime}$ acts trivially on $A_{0}$ and (1) holds.

The subgroup $\Gamma_{0}:=C_{\Gamma}\left(A / A_{0}\right)$ has finite index in $\Gamma$. On the other hand, by the above, $\Sigma:=\Gamma^{\prime} \cap \Gamma_{0}$ stabilizes the series $0 \leq A_{0} \leq A$ and embeds in $\operatorname{Hom}\left(A / A_{0}, A_{0}\right)$, which is bounded by $m:=\left|A / A_{0}\right|$. Thus $[A, \Sigma] \leq A_{0}[m]=B[m] \oplus C[m] \oplus D[m]$. As each $\gamma \in \Gamma$ acts by multiplications on $B[\mathrm{~m}], C[\mathrm{~m}], D[\mathrm{~m}]$ and $\Gamma$ is finitely generated, $\left|\Gamma / \Gamma_{2}\right|$ is finite, where $\Gamma_{2}=C_{\Gamma_{0}}([A, \Sigma])$. On the other hand, we have that $\left[A, \Sigma, \Gamma_{2}\right]=0$ and $\left[A, \Gamma_{2}, \Sigma\right] \leq\left[A_{0}, \Sigma\right]=0$. Thus, by the Three Subgroup Lemma $\left[\Gamma_{2}, \Sigma, A\right]=0$, that is $\Sigma$ is contained in the center of $\Gamma_{2}$ which turns to be nilpotent and finitely generated as well. Therefore, $\Gamma$ has the maximal condition on subgroups and $\Gamma^{\prime}$ is finite, being periodic. Finally, as $\Gamma$ is finitely generated, we have $\Gamma$ is central-by-finite.

Proof of Corollary B In part (1), by Propositions 2.2 and 2.3, LIN implies inertial. Also, in any case, if $\varphi$ is invertible, then $\varphi$ is $\operatorname{LIN}$ iff $\varphi^{-1}$ is inertial.

For statement (2), note that if $\gamma_{1}$ is inertial and $\gamma_{2}$ is LIN, then $\gamma_{1} \gamma_{2}=\gamma_{2} \gamma_{1}\left[\gamma_{1}, \gamma_{2}\right]$ where $\gamma_{1}\left[\gamma_{1}, \gamma_{2}\right]$ is inertial as $\left[\gamma_{1}, \gamma_{2}\right]$ is finitary.

Proof of Proposition $A$ Let $A$ be an abelian group and $T:=T(A)$. Suppose $V$ is a torsion free subgroup such that $A / V$ is periodic and denote $\bar{A}:=A /(V+T)$, then the stabilizer $\Sigma$ of the series $0 \leq(V+T) \leq A$ is canonically isomorphic to $\operatorname{Hom}(\bar{A}, V+T)=\operatorname{Hom}(\bar{A}, T)$. In the particular case when $V$ has finite rank and $A / V$ is locally cyclic, then by Proposition 2.3 we have $\Sigma \leq I A u t(A)$ and $\Sigma \cap F A u t(A)$ corresponds to the subgroup of $\operatorname{Hom}(\bar{A}, T)$ formed by the homomorphisms with finite image. Moreover, if in addition, for each prime 
$p, A_{p}$ has order $p$ while the $p$-component of $A / V$ has finite order (at least) $p^{2}$, we have: $\Sigma \simeq \operatorname{Hom}(\bar{A}, T) \simeq \prod_{p} \mathbb{Z}(p)$ and $\Sigma \cap F A u t(A) \simeq \bigoplus_{p} \mathbb{Z}(p)$, where $p$ ranges over the set of all primes.

To show the existence of a group $A$ as above, let $G:=B \oplus C$ where $B:=\prod_{p}\left\langle b_{p}\right\rangle$, $C:=\prod_{p}\left\langle c_{p}\right\rangle$, and $b_{p}, c_{p}$ have order $p, p^{2}$, respectively. Consider the (aperiodic) element $v:=\left(b_{p}+p c_{p}\right)_{p} \in G$ and $V:=\langle v\rangle$. Note that for each prime $p$ there exists an element $d_{(p)} \in G$ such that $p d_{(p)}=v-b_{p}$. Define $A:=V+\left\langle d_{(p)} \mid p\right\rangle$. Then, we have that $A / T \simeq\langle 1 / p \mid p\rangle \leq \mathbb{Q}$ as it has torsion-free rank 1 and $v+T$ has $p$-height 1 for each prime. Then, $T=T(B) \simeq \bigoplus_{p} \mathbb{Z}(p)$, while the $p$-component of $A / V$ is generated by $d_{(p)}+V$ and has order $p^{2}$ as $p d_{(p)}=v-b_{p}$.

\section{References}

1. Belyaev, V.V., Kuzucuoglu, M., Seckin, E.: Totally inert groups. Rend. Semin. Mat. Univ. Padova 102, 151-156 (1999)

2. Casolo, C.: Groups with finite conjugacy classes of subnormal subgroups. Rend. Semin. Mat. Univ. Padova 81, 107-149 (1989)

3. Dardano, U., Rinauro, S.: Inertial automorphisms of an abelian group. Rend. Semin. Mat. Univ. Padova 127, 213-233 (2012)

4. Dardano, U., Rinauro, S.: On the ring of inertial endomorphisms of an abelian group. Ricerche Mat. (2014). doi:10.1007/s11587-014-0199-3

5. De Falco, M., de Giovanni, F., Musella, C., Trabelsi, N.: Strongly inertial groups. Commun. Algebra 41, 2213-2227 (2013)

6. Dikranjan, D., Giordano Bruno, A., Salce, L., Virili, S.: Fully inert subgroups of divisible Abelian groups. J. Group Theory 16, 915-939 (2013)

7. Dikranjan, D., Giordano Bruno, A., Salce, L., Virili, S.: Intrinsic algebraic entropy. J. Pure Appl. Algebra (2014). doi:10.1016/j.jpaa.2014.09.033

8. Dixon, M., Evans, M.J., Tortora, A.: On totally inert simple groups. Cent. Eur. J. Math. 8(1), 22-25 (2010)

9. Franciosi, S., de Giovanni, F., Newell, M.L.: Groups whose subnormal subgroups are normal-by-finite. Commun. Alg. 23(14), 5483-5497 (1995)

10. Fuchs, L.: Infinite Abelian Groups. Academic Press, New York (1970-1973)

11. Robinson, D.J.S.: On inert subgroups of a group. Rend. Semin. Mat. Univ. Padova 115, 137-159 (2006)

12. Specht, W., Heineken, H.: Gruppen mit endlicher Komponentenzahl fastgleicher Untergruppen. Math. Nachr. 134, 73-82 (1987)

13. Wehrfritz, B.A.F.: Finite-finitary groups of automorphisms. J. Algebra Appl. 1(4), 375-389 (2002) 